\title{
How engineering students describe three-dimensional forces
}

\author{
Janice Miller-Young, Ph.D., P.Eng. \\ Associate Professor, Mount Royal University \\ jmyoung@mtroyal.ca
}

\begin{abstract}
The purpose of this study was to find out how students visualize three-dimensional statics problems from twodimensional diagrams early in a first-year engineering course. Think-alouds were conducted where students were asked to describe magnitudes and directions of various forces acting in a three-dimensional space. An unanticipated result was that students solved for components and direction cosines of forces, rather than directly answering the questions. Students had the most trouble visualizing points and vectors that were behind the plane of the page.
\end{abstract}

\section{Introduction}

Spatial visualization has been defined as "the ability to mentally restructure or manipulate the components of the visual stimulus" [1]. Student spatial skills are not only important for being able to visualize structures in mechanics, but are considered to be one of the most important outcomes for engineering students [2] and are directly linked to future success in their professional work [3]. In chemistry, visualization ability has even been related to problem solving skills in areas where the problem does not actually involve visualization [4].

Numerous studies have indicated that spatial abilities can be improved through the use of spatial exercises, drafting instruction, physical models and computer models (eg. [5], [6]). Sorby and Baartmans [7] speculated that a diverse range of spatial activities may be the key to effective spatial ability. In fact, simply taking a physics course has been shown to improve visual-spatial abilities [8].

In my experience, one of the difficulties students have in a first-year engineering mechanics course is learning to interpret and analyze three-dimensional (3D) static equilibrium problems. 3D structures and forces are typically presented in two-dimensional (2D) drawings from a textbook, and some students seem to be able to visualize the structures and forces quickly while others struggle. In order to increase my effectiveness as a teacher, I wanted to know exactly how students were visualizing these problems early in their learning, and what aspects were giving them trouble.

Think-alouds are a type of verbal protocol and are a mainstay in cognitive psychology. They are used to infer mental models by observing students while they are actually engaged in mental activities. The subjects are asked to "think aloud" while engaged in a task which could normally be carried out alone [9]. Since it is assumed that the majority of students represent a very limited number of distinctively different ways of understanding, small sample sizes are usually considered appropriate [10].

This research project took place in the researcher's first-year, first semester mechanics course at Mount Royal University. The class size was 40 students, with tutorials of 20 students, also taught by the researcher. Mechanics I is generally considered to be the most difficult first-year course, and has high withdrawal and failure rates. In fact, in the fall 2009 semester when this study ran, $50 \%$ of the students withdrew before the withdrawal date, and of the remaining students only $50 \%$ achieved a high enough mark (C- or better) to take courses that have Mechanics I as a prerequisite. In other words, only $25 \%$ of the students who started the semester successfully completed the course.

Think-alouds were used in this study to answer the question: What is the process that students in a firstyear engineering class go through in visualizing 3D statics problems from 2D drawings?

\section{Methods}

\subsection{Participant recruitment}

The participants were recruited at the beginning of the semester by an independent faculty member and consent forms were held until the final marks for the course were submitted, so that the instructor/ researcher did not know who had agreed to participate in the study until after the course was finished. 


\subsection{Think-alouds}

Think-aloud sessions were conducted one to two weeks after the concepts of three-dimensional forces, unit vectors and direction cosines were introduced in the class. The sessions were conducted by a faculty member outside of engineering to ensure the students would feel comfortable during the session and to keep the participants' identity unknown to the instructor until the end of the course.

Participants were given standard instructions for the think-aloud task. First, they completed a simple warm-up activity to practice verbalizing their thinking process. When the participant had completed this warm-up and had no further questions, they were asked to answer two additional problems. For each question presented, they were instructed to read the question out loud and then to speak out loud what they were thinking as they worked through the questions, and not to try to explain or summarize until the end.

The first question was the following:

a) Please describe the direction and magnitude of the forces in the diagram below (whether they are into/out of the page, which one is larger, and which one has larger $\mathrm{x}, \mathrm{y}$ and $\mathrm{z}$ components).

b) Explain how you visualized this problem and arrived at your answer.

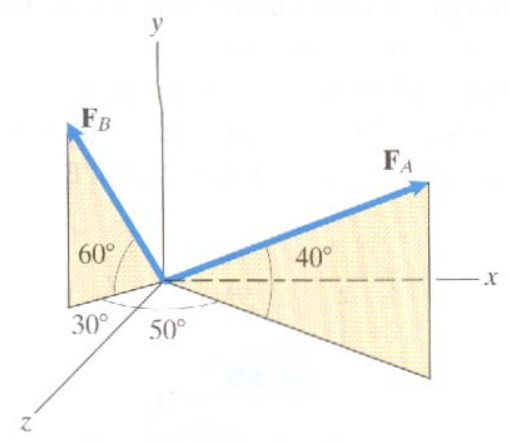

Figure 1. Question 1 from the think-aloud.

The second question was the following:

a) Please describe the direction of the forces in the diagrams below (whether they go into/out of the page). b) Write the 3-D co-ordinates of points A, B, C and D.

c) Explain how you visualized this problem and arrived at your answers.

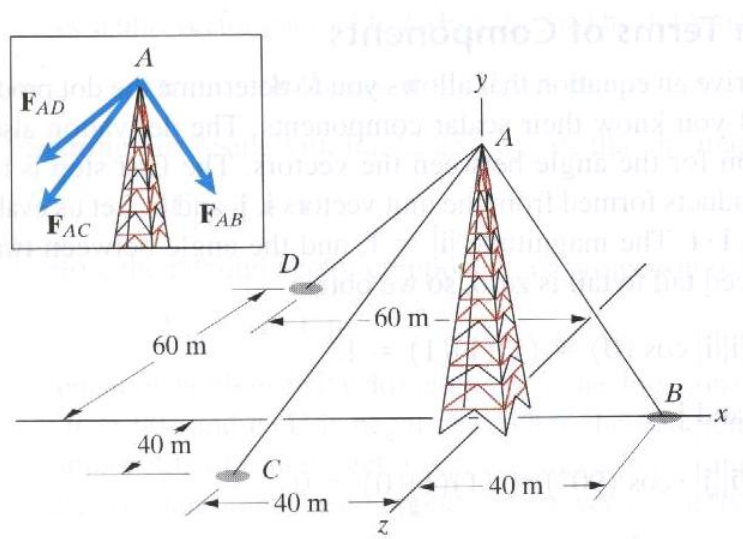

Figure 2. Question 2 from the think-aloud.

The only intervention made by the interviewer during the think-aloud was to remind participants to think out loud if they appeared to be thinking but had stopped talking. After each question was completed the interviewer asked "Can you summarize how you arrived at your answer?" if the participant had not already done so.

Think aloud protocols were audio recorded and any paper the students wrote on during the think-aloud was also kept for analysis by the researcher. Recordings were transcribed by the researcher after the course was finished.

This study was approved by the Human Research Ethics Board of Mount Royal University.

\section{Results}

\subsection{Participants}

Ten students from the fall 2009 semester of a firstyear mechanics course volunteered for the study. Of these ten, five attended the think-aloud sessions, which took place outside of class time. These five participants represent a range of students in terms of course performance, with Subjects A and B achieving A- in the class, Subject $C$ achieving a C, and Subjects $\mathrm{D}$ and $\mathrm{E}$ failing the first midterm and ultimately withdrawing from the course before the final exam. 
Table 1. Student participants

\begin{tabular}{|c|c|c|c|}
\hline Participant & $\begin{array}{c}\text { Midterm } \\
\text { 1 mark } \\
\text { (\%) }\end{array}$ & $\begin{array}{c}\text { Final } \\
\text { grade }\end{array}$ & $\begin{array}{c}\text { Performance } \\
\text { classification }\end{array}$ \\
\hline A & 91 & A- & High \\
\hline B & 86 & A- & High \\
\hline C & 91 & C & Avg \\
\hline D & 45 & N/A & Low \\
\hline E & 36 & N/A & Low \\
\hline
\end{tabular}

The think-aloud transcriptions are currently being coded and analyzed for themes. Selected results are presented here.

\subsection{Equations rather than answers}

The most interesting result was that none of the five students answered the questions as anticipated. In fact, they did not directly answer parts of the questions. In short, they tried to calculate the directions and magnitudes of the vectors rather than describe them, even when there was not enough information to calculate an answer.

Subject $\boldsymbol{A}$ treated the think-aloud questions as if they were assignment questions and wrote out things that are typically asked for on an assignment but that the researcher had not intended to be done in this case (eg. summarize the given information and what the question asks for). The participant promptly set about calculating equations, and did not pause for any amount of time when interpreting the diagram. His answer, both orally and written, to question 1 was:

$$
\begin{array}{ll}
\text { “F } F_{x}=F_{B} \cos 60 \cos 30 & \theta_{x}=\cos ^{-1}\left(F_{y} / F_{B}\right) \\
F_{y}=F_{B} \sin 60 & \theta_{y}=\cos ^{-1}\left(F_{x} / F_{B}\right)>90^{\circ} \\
F_{z}=F_{B} \cos 60 \sin 60 & \theta_{z}=\cos ^{-1}\left(F_{z} / F_{B}\right) "
\end{array}
$$

While the above mathematical statements are mostly correct ( $F_{x}$ should be negative), they do not actually answer the questions asked unless the magnitudes of the forces are given. The student felt that he had answered the question sufficiently.

Subject $\boldsymbol{D}$ was not able to answer question 1 of the think-aloud. He was pre-occupied with not being able to remember the equations (although the equations are simple and can be derived from trigonometry easily if the diagram can be visualized properly). Subject D repeatedly said things like:

$$
\begin{aligned}
& \text { "I'm not quite sure what the formula is..." } \\
& \text { "I'm trying to remember the formula..." }
\end{aligned}
$$

"I can't exactly remember how to do [it], but anyways that's how I'd go through it and do that one."

Subject D did not give any written answer to question 1.

Subject $\boldsymbol{E}$ took more time but more or less ended up with the same answer as Subject A for question 1 . This subject experienced some confusion between sine and cosine. He said things like:

"For $F_{A}$, like the magnitude of it, you can just write the equation for it."

"the opposite would be the y coordinate of it, so it's cos.... Wait, this should be sine..."

"so which one's larger... ok so those are the magnitudes of each of the forces."

"Direction of $F_{A}=$ positive $x, y$, negative $z$ "

"Direction of $F_{B}=$ negative $x$, but positive $y, z$ "

Subject E's answers are correct except for the zcomponent of $\mathrm{F}_{\mathrm{A}}$. This participant came closer to answering the actual question than the participants above, although he still did not specifically say whether the forces went into or out of the page, nor did he say which forces and which components of the forces were larger.

The other students all gave similar answers to both questions in terms of writing equations but not answering the questions, although some struggled more than others with the fact that all the necessary information to calculate an answer was not given, and some students took longer to visualize/calculate than others.

\subsection{Visualization bottlenecks}

The second major theme was that students had the most trouble visualizing vectors and identifying points that were behind the plane of the page.

Subject $\boldsymbol{C}$ initially had difficulty visualizing point $\mathrm{D}$ in question 2 and took a very long time before he said:

"FofD...... you can't really tell which quadrant it goes in..... it looks like it stops over in quadrant 4... so FofD is going into the page."

Subject D initially made the statement:

"it looks like point D is floating up here..." 
but eventually wrote the co-ordinates of point $\mathrm{D}$ in question 2 correctly and stated that $\mathrm{F}_{\mathrm{D}}$ was going into the page.

Subject $\boldsymbol{E}$ gave the co-ordinates of point D in question 2 to be $(-60, y,-60)$ as if the height of that point is unknown, which it is not.

\section{Discussion}

While the way participants responded was unanticipated, their responses did in fact serve to answer the research question and were very illuminating for the researcher as an instructor.

First, the way participants responded to the questions (providing equations but generally not answering the questions) supports previous results that show how expectation schemas can have a big impact in how students respond [11]. Since the students in the current study were primarily tested in class on their calculations, in hindsight it is not surprising that their expectations of what they were going to be asked to do precluded the participants from answering the question. Redish et al. [12] showed that student attitudes and expectations that "memorizing equations is important" and "any numbers a student needs will always be given to them" can actually increase as a result of traditional instruction in introductory physics for engineers. However, the fact that the think-alouds in this current study were conducted in week 3 and 4 of a first-year, first-semester course demonstrates that it is important to begin dispelling these misconceptions very early in the students' postsecondary academic career, if not before. The instructor will make a point of using more word problems and concept-based problems as early as possible in this course in the future.

It has also been argued that, in the absence of deep learning, students resort to learning surface routines and language in the hope that they can pass these off as real understanding [13]. It is not possible to tell from the students' responses in this study whether they didn't answer the questions because of their expectation of what was going to be asked, or their lack of realization that the problem could be answered without doing numerical calculations. In any case, the students did seem to feel that they had answered the question as correctly as possible with the quantitative information given. This result supports the concern in the current educational literature that students' ability to solve problems does not necessarily demonstrate that they have a conceptual understanding [14], [15], [16].

Finally, for the instructor, this study helped to "reverse engineer" the process students go through in learning to visualize these types of problems. In other words, it is difficult as an expert in a subject to "decompile" the knowledge that is used in thinking about a problem because the knowledge feels innate. This makes it hard for the instructor/expert to understand the students' difficulties when learning to solve the same problem. In this study, the students used analytic processing to answer the problem, in that they broke the problem down into individual parts, rather than gestalt processing, where an image is transformed and organized as a whole [17], [18], as the instructor would have done. Their analytic processing made the components of problems that gave them trouble very "visible" to the instructor. For example, the instructor knew that some students had difficulty with using sines and cosines at the beginning of the mechanics course, but was unaware that they had difficulty "seeing" points and vectors that were behind the plane of the page. This result will also influence how such problems will be presented to students in future classes.

Meyer and Land [19] propose that studentcentered teaching has an element of responsiveness that is sensitive to variation in the manner in which students engage with the context and content of their learning. Thus, in order to be responsive, the variation in student learning must be externalised in a form that can be responded to. Similarly, Land et al. [20] argue that "we can't second guess where students are coming from or what their uncertainties are". The think-alouds in this study were very helpful for the instructor as they made visible student uncertainties and misconceptions that the instructor would otherwise have been unaware of. While there are ethical and organizational difficulties in performing think-alouds with a researcher's own students, in this instructor's opinion, the difficulties were more than worth the effort considering the results achieved.

\section{References}

[1] M.G. McGee, Human Spatial Abilities, Sources of Sex Differences, Praeger, New York, 1979.

[2] M. Alias, T.R. Black, and D.E. Gray, "Effects of instructions on Spatial Visualisation Ability in Civil Engineering Students”, International Education Journal, 2002, pp 1-12.

[3] S. Hsi, M.C. Linn, and J.E. Bell, "The role of spatial reasoning in engineering and the design of spatial instruction”, Journal of Engineering Education 1997, pp. 151-58. 
[4] G.M. Bodner and R.B. Guay, "The Purdue Visualization of Rotations Test", The Chemical Educator,1997, pp. 1-17.

[5] S.E. Wiley, "Computer graphics and the development of visual perception in engineering graphics curricula”, Engineering Design Graphics Journal, 1990, pp. 30-35.

[6] C. Miller, "Enhancing spatial visualization abilities through the use of real and computer generated models”, Proceedings of the 1990 Annual conference for American Society of Engineering Education, 1990.

[7] S.A. Sorby and B.G. Baartmans, "A course for the development of 3D spatial visualization skills", Engineering Design Graphics Journal , 1996, pp. 1320.

[8] G.J. Pallrand and F. Seeber, "Spatial ability and achievement in introductory physics", Journal of Research in Science Teaching, 1984, pp. 507-516.

[9] R.A. Streveler, T.A. Litzinger, R.L. Miller, and P.S. Steif, "Learning Conceptual Knowledge in the Engineering Sciences: Overview and Future Research Directions”, Journal of Engineering Education, 2008, pp. 279-94.

[10] F. Marton, On the structure of awareness. In Phenomenographic research: Variations in method, Royal Melbourne Institute of Technology, Melbourne, Australia, 1994.

[11] E.F. Redish and K.A. Smith, "Looking beyond content: Skill development for engineers", Journal of Engineering Education, 2008, pp. 295-307.

[12] E.F. Redish, J.M. Saul, and R.N. Steinberg, "Student expectations in introductory physics", American Journal of Physics, 1998, pp. 212-224.
[13] P. Davies, “Threshold concepts: How can we recognise them?”, Biennial Conference of the European Association for Research into Learning and Instruction, Padua, Italy, 2003.

[14] I.A. Halloun and D. Hestenes, "The initial knowledge state of college physics students", American Journal of Physics, 1985, pp. 1043-1055.

[15] P. Davies and J. Mangan, "Threshold concepts and the integration of understanding in economics", Studies in Higher Education, 2007, pp. 711-726.

[16] D. Montfort, An investigation of students' conceptual understanding in related sophomore to graduate-level engineering and mechanics courses, Ph.D. Thesis, Washington State University, 2007.

[17] I.M. Smith, Spatial Ability, Its Educational and Social Significance, Robert R. Knapp, San Diego, CA, 1964.

[18] C. Spearman and L. Jones, Human Abilities, Macmillan, London, 1950.

[19] J.H.F. Meyer and R. Land, "Threshold concepts and troublesome knowledge (2): Epistemological considerations and a conceptual framework for teaching and learning”, Higher Education, 2005, pp. 373-388.

[20] R. Land, G. Cousin, J.H.F. Meyer, and P. Davies, "Threshold concepts and troublesome knowledge (3): implications for course design and evaluation”, Improving Student Learning Diversity and Inclusivity, Oxford, 2005. 